\title{
Structural Analysis of a Melaminium Polyphosphate from X-ray Powder Diffraction and Solid-State NMR Data
}

\author{
Vladimir Brodski,* René Peschar, and Henk Schenk \\ Universiteit van Amsterdam, van't Hoff Institute for Molecular Sciences, Laboratory for Crystallography, \\ Nieuwe Achtergracht 166, 1018WV Amsterdam, The Netherlands

\begin{abstract}
Andreas Brinkmann, Tom G. Bloemberg, Ernst R. H. van Eck, and Arno P. M. Kentgens
Radboud University Nijmegen, Physical Chemistry / Solid State NMR, Institute for Molecules and Materials, Toernooiveld 1, 6525 ED Nijmegen, The Netherlands
\end{abstract}

Received: March 11, 2005; In Final Form: May 10, 2005

\begin{abstract}
The crystal structure of the environmentally friendly flame retardant melaminium polyphosphate (MPoly) (2,4,6-triamino-1,3,5-triazinium $\left.\cdot \mathrm{PO}_{3}\right)_{n}$ was determined by a direct-space global optimization technique from X-ray powder diffraction data. Solid-state NMR was used to corroborate the proposed hydrogen-bonding model and to determine the average degree of polymerization $(n>100)$. An analysis of the crystal structure of MPoly reveals aspects of molecular geometry and packing that are characteristic for melamine-containing compounds and polyphosphate salts. A comparison of MPoly with the crystal structures of its precursors melaminium orthophosphate (MP) and melaminium dihydrogenpyrophosphate (MPy) provides insight in the mechanism of the endothermic dehydration processes that takes place in the reaction path MP $\rightarrow$ MPy $\rightarrow$ MPoly. Solid-state NMR characterization of various samples of the same batch showed inhomogeneities in the MPoly composition. Various quantities of orthophosphates were found, which cannot be assigned to be MP.
\end{abstract}

\section{Introduction}

Most polymers that are widely used in industry and consumer products are not intrinsically flame-retardant and burn completely after catching fire. An efficient and cheap way to reduce their flammability is to admix flame retardants. During the past years, a large interest has arisen in melamine-phosphate-based flame retardants (MPBFRs). ${ }^{1-8}$ These nonhazardous and environmentally friendly materials are considered to be an attractive alternative to halogen-containing flame retardants. The latter release toxic and corrosive gases during combustion and are increasingly banned by more stringent legislation for that reason.

MPBFRs are salts of melamine and a phosphate, either orthophosphate or condensed forms of phosphate, such as pyrophosphate and polyphosphate that result from a thermal condensation. ${ }^{1}$ Often in these compounds, the melamine moiety is a cation (melaminium) with an endocyclic nitrogen being protonated because of hydrogen transfer from a phosphate.

MPBFRs are polycrystalline materials with high thermal stability and very low solubility in water and organic solvents. ${ }^{2,3}$ They are admixed in many industrial and consumer products that require protection against fire, for example, plastics ${ }^{1,6}$ and coatings. ${ }^{3}$ MPBFRs are active in the condensed phase: During combustion, an organic-inorganic porous char layer is formed at the surface of the polymer that shields off the polymer from heat, air, and pyrolysis products. Although phosphoric acid shows catalytic activity and melamine is involved in the intumescence process ("swelling") that leads to the char layer as a blowing agent, the precise flame-retardant mechanism of MPBFRs and the role of the individual components therein have not been clarified completely.

\footnotetext{
*brodski@science.uva.nl.
}

Flame-retardant activity of MPBFRs such as melaminium orthophosphate (MP), ${ }^{1-3,9}$ melaminium dihydrogenpyrophosphate $(\mathrm{MPy})^{1-3,8,9}$ and melaminium polyphosphate (MPoly) $)^{1,2,5}$ is well-established. The polycondensation process from MP via MPy to MPoly that influences the flame-retardant activity, ${ }^{3}$ does not seem to have been studied in detail from a structural point of view, mainly because of the polycrystalline nature of the materials. In recent years, however, considerable progress has been made in solving crystal structures from X-ray powder diffraction (XRPD) data using direct-space search methodology (see ref 10 and references therein). In addition, solid-state NMR has proven to be well-suited to investigate hydrogen-bonded systems such as benzoxazine dimers and oligomers. ${ }^{11-14}$ Recently, we elucidated the crystal structures of the compounds $\mathrm{MP}^{15}$ and $\mathrm{MPy}^{16}$ using the above-mentioned techniques and density-functional calculations. Here, we report the structure of the melamine polyphosphate MPoly (degree of polymerization > 100).

To establish common packing characteristics and aspects of molecular geometry, the crystal structure of MPoly is compared with those of MP, MPy, other melamine-containing compounds, and polyphosphate salts. An analysis of the crystal-structure models of MPoly, MPy, and MP delivers detailed information concerning the condensation reaction route $\mathrm{MP} \rightarrow \mathrm{MPy} \rightarrow$ MPoly that is important in understanding the flame-retardant activity. ${ }^{1}$

\section{Experimental Section}

Synthesis. The samples were obtained from DSM who manufactured the material in accordance with their industrial procedures: The synthesis of MPy was carried out as discussed 
in ref 16. MPoly (white crystalline powder) was synthesized through thermal curing of MPy for $3 \mathrm{~h}$ at $280^{\circ} \mathrm{C}$, which resulted in a batch of $0.5 \mathrm{~kg}$ MPoly from which various samples were taken. The samples originating from this batch showed significantly different properties. Solid-state NMR established the degree of polymerization to be in the range $100-170$, but to be at least $\sim 100$ in all cases, and showed the presence of a large amount (up to 24\%) of orthophosphate impurities. With XRPD, a minor crystalline phase was detected, but the amount varied from sample to sample. Despite the significant spread in the degree of polymerization and the orthophosphate impurity level, all samples contained the same crystalline phase of MPoly. The differences between the samples are attributed to an inhomogeneous batch of MPoly due to inhomogeneous heat transfer and insufficient mixing during the synthesis.

Crystallographic Methods. Single crystals of MPoly could not be grown, so the crystal structure had to be determined on the basis of XRPD data. An XRPD recording was made at room temperature with an Enraf-Nonius FR 552 Guinier Johansson camera equipped with a Johansson monochromator ${ }^{17}$ and using $\mathrm{Cu} K \alpha_{1}$ radiation $(\lambda=1.54060 \AA)$. The sample was prepared by pressing the powder into a thin layer onto Mylar foil. During data collection, the sample was rotated to improve particle statistics. For indexing, ${ }^{18}$ the accurate positions of 60 lines in the interval $2 \theta=0.0-42.0^{\circ}$ were read off from the Guinier photograph. For the structure determination and Rietveld refinement, the Guinier photograph was read out with an optical instrument and, using a Johansson LS-18 microdensimeter, digitized to a pattern with a step size of $0.01^{\circ} 2 \theta$.

An initial molecular model of MPoly was built, consisting of two melamine moieties and one $\mathrm{P}_{2} \mathrm{O}_{7}$ moiety in their respective geometries in MPy. ${ }^{16}$ To position the moieties in the asymmetric part of the unit cell, the Monte Carlo code Organa ${ }^{19}$ was used. Only non-hydrogen atoms were considered at this stage.

The crystal structure solution obtained with the Monte Carlo calculations was used as the starting model in a Rietveld refinement using the program $G S A S .{ }^{20}$ The peaks in the range $2 \theta=6.49-80.35^{\circ}$ were fitted with a split-type pseudo-Voigt peak-profile function. ${ }^{21,22}$ The background was modeled by a Chebyshev polynomial of the first kind with 14 parameters. The preferred orientation was described with a spherical harmonics function. ${ }^{23}$ The isotropic displacement parameters $U_{\text {iso }}$ of identical atom types were coupled, and a small damping was applied during all refinement stages. The $U_{\text {iso }}$ values of hydrogen atoms were not refined.

To avoid distortion of the model, in the refinement process, each bond length and bond angle in the molecule were restrained using an ideal value and an associated standard unit (s.u.) (the function that was minimized during the restrained refinement is defined in expression 1 of ref 24). Distance and angle restraints were obtained by averaging the corresponding quantities in the structures of polyphosphate salts, ${ }^{25-31} \mathrm{MP},{ }^{15} \mathrm{MPy},{ }^{16}$ and other compounds with a positively charged $(+1)$ melamine moiety as present in the Cambridge Structural Database. ${ }^{32}$ The non-hydrogen atom s.u.'s of the restraints were taken as $1 \%$ and 5\% for bond distances and bond angles, respectively. For hydrogen atoms, the respective s.u.'s were taken as $0.01 \%$ and $0.05 \%$. The initial, strong restraints (weighting factor $f=1000$, expression 1 of ref 24) were gradually reduced to soft restraints $(f=5)$ in the final refinement.

Solid-State NMR Spectroscopy. Single-pulse ${ }^{1} \mathrm{H}$ magicangle-spinning (MAS) solid-state NMR spectra of MP, MPy, and MPoly were obtained at a static field of $18.8 \mathrm{~T}$ (Varian
Inova) and sample-spinning frequency of $49.0 \mathrm{kHz}$ using a home-built probehead based on a Samoson ${ }^{33}$-type MAS assembly. The ${ }^{31} \mathrm{P}$ cross-polarization magic-angle-spinning (CP-MAS) spectra were acquired at a static field of $7.05 \mathrm{~T}$ (Chemagnetics Infinity) and sample-spinning frequencies of $4.0 \mathrm{kHz}$ and $12.0 \mathrm{kHz}$ using a $\mathrm{CP}$ contact time of $4 \mathrm{~ms}$. Continuous-wave decoupling with ${ }^{1} \mathrm{H}$ nutation frequency of $100 \mathrm{kHz}$ was used during acquisition at $4 \mathrm{kHz}$ spinning, while two-pulse phase modulation (TPPM) decoupling ${ }^{34}$ with a ${ }^{1} \mathrm{H}$ nutation frequency of $100 \mathrm{kHz}$, pulse durations of $4.8 \mu \mathrm{s}$, and radio frequency phases of $\pm 12^{\circ}$ was used during acquisition at $12 \mathrm{kHz}$ spinning. The two-dimensional ${ }^{1} \mathrm{H}-{ }^{31} \mathrm{P}$ heteronuclear correlation spectrum of MPoly and MP were obtained at a static field of $7.05 \mathrm{~T}$ and a sample-spinning frequency of $12.0 \mathrm{kHz}$. Frequency-switched Lee-Goldburg homonuclear decoupling ${ }^{35}$ with a nutation frequency of $100 \mathrm{kHz}$, frequency jumps of $\pm 70.711 \mathrm{kHz}$, and an overall offset of $-1.5 \mathrm{kHz}$ was used in the indirect ${ }^{1} \mathrm{H}$ dimension. The ${ }^{1} \mathrm{H}$ magnetization was transferred to ${ }^{31} \mathrm{P}$ using Lee-Goldburg cross-polarization ${ }^{36}$ with a duration of $0.2 \mathrm{~ms}$. TPPM decoupling with a ${ }^{1} \mathrm{H}$ nutation frequency of $100 \mathrm{kHz}$ was applied during acquisition. The pulse durations and radio frequency pulses for TPPM were given by $(5 \mu \mathrm{s}$, $\left.\pm 30^{\circ}\right)$ and $\left(5.4 \mu \mathrm{s}, \pm 15^{\circ}\right)$ for the MPoly and MP sample, respectively. The ${ }^{31} \mathrm{P}$ single-pulse spectra of two samples of MPoly were acquired at a static field of $9.4 \mathrm{~T}$ using a spinning frequency of $12 \mathrm{kHz}$. In both cases, proton decoupling with a nutation frequency of $56 \mathrm{kHz}$ was used during acquisition. The ${ }^{31} \mathrm{P} T_{1}$ measurements were performed under the same experimental conditions by a ${ }^{31} \mathrm{P}$ saturation recovery MAS experiment.

\section{Results and Discussion}

X-ray Analysis and Structure Determination of MPoly. The indexing of the XRPD pattern by the program ITO ${ }^{18}$ yielded a monoclinic unit cell (Table 1) and two possible space groups, $C 2 / c$ or $C c$. Despite the high indexing figure of merit $\left(M_{20}=\right.$ 62 ), a number of weak lines could not be indexed, indicating the presence of another minor crystalline phase. The pattern of a second sample from the same MPoly batch revealed the same peak positions and relative intensities for the main crystalline phase, suggesting that the same MPoly has been formed during the polycondensation. The peak positions of the nonindexed peaks in both samples were the same. However, the relative intensities of the nonindexed peaks in the second sample appeared to be $\sim 4$ times larger than those in the first sample. For this reason, the first sample has been chosen for further analysis.

For structure determination, the space group $C c$ was chosen, because its symmetry elements are a subset of those of $C 2 / c$. In accordance with this choice, 2 melamine moieties and $1 \mathrm{P}_{2} \mathrm{O}_{6}$ fragment of an infinite chain of phosphates are expected in the asymmetric part of the unit cell. The calculated density $\left(d_{\mathrm{c}}=1.78 \mathrm{~g} \mathrm{~cm}^{-3}\right)$ corresponds reasonably well with the experimental density $\left(d_{\mathrm{m}}=1.82 \mathrm{~g} \mathrm{~cm}^{-3}\right)$. At the first stage of the structure determination, the $\mathrm{P}_{2} \mathrm{O}_{7}$ moiety was considered (see Experimental Section). When the $R_{\mathrm{W}}$ factor dropped below $30 \%$ during the Monte Carlo run and a preliminary model of MPoly was obtained, an analysis pointed out that one of the oxygen atoms should be omitted from the $\mathrm{P}_{2} \mathrm{O}_{7}$ moiety, and the calculation was continued with the $\mathrm{P}_{2} \mathrm{O}_{6}$. Finally, the calculations were stopped when the $R_{\mathrm{w}}$ factor became $\sim 10 \%$ for 101 low-angle reflections (resolution $\approx 2.0 \AA$ ).

After finishing the refinement in $C c$, a check for possible missing symmetry indicated that the symmetry should be changed to $C 2 / c$. After this, a final refinement has been carried out in $C 2 / c$. 
TABLE 1: Crystallographic Data of MPoly

\begin{tabular}{|c|c|c|c|}
\hline \multicolumn{2}{|c|}{ crystal data } & \multicolumn{2}{|c|}{ data collection and refinement } \\
\hline formula & $\overline{\mathrm{C}_{3} \mathrm{H}_{7} \mathrm{~N}_{6}{ }^{+} \cdot \mathrm{PO}_{3}{ }^{-}}$ & data range & $6.49 \leq 2 \theta \leq 80.35^{\circ}$ \\
\hline crystal system & monoclinic & no. of data points & 7635 \\
\hline space group & $C 2 / c$ (no. 15$)$ & no. of reflections & 1012 \\
\hline$Z$ & 8 & temperature $(\mathrm{K})$ & 294 \\
\hline $\mathrm{A}(\AA)$ & $15.9490(4)$ & GoF & 6.22 \\
\hline $\mathrm{B}(\AA)$ & $4.37243(10)$ & $R_{\mathrm{p}}(\%)$ & $6.82(6.06)^{a}$ \\
\hline $\mathrm{C}(\AA)$ & $22.2410(5)$ & $R_{\mathrm{wp}}(\%)$ & $9.00(7.48)^{a}$ \\
\hline$\alpha\left(^{\circ}\right)$ & 90. & no. of refined parameters & \\
\hline$\beta\left(^{\circ}\right)$ & 98.9963(15) & lattice & 4 \\
\hline$\gamma\left(\left(^{\circ}\right)\right.$ & 90. & positional & 60 \\
\hline$V\left(\AA^{3}\right)$ & $1531.79(6)$ & thermal & 4 \\
\hline \multirow{4}{*}{$d_{\mathrm{m}} / d_{\mathrm{c}}\left(\mathrm{g} \mathrm{cm}^{-3}\right)$} & $1.82 / 1.78$ & texture & 15 \\
\hline & & Profile & 10 \\
\hline & & Background & 14 \\
\hline & & zero-shift & 1 \\
\hline
\end{tabular}

${ }^{a}$ In parentheses, values of $R$ factors with subtracted background are given.

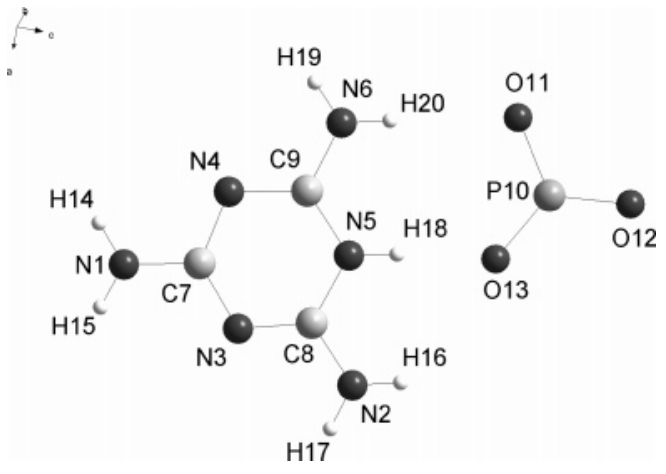

Figure 1. Labeling of the atoms in the asymmetric part of the unit cell of MPoly.

In addition to the hydrogens bonded to the exocyclic nitrogens, assumed to be in the usual planar $-\mathrm{NH}_{2}$ conformation, one other hydrogen also had to be positioned in $C 2 / c$ in order to neutralize the charge. One of the endocyclic nitrogens of the melamine moiety has a short contact with an oxygen atom of the polyphosphate, suggesting a hydrogen bond. A comparison of the crystal structure of MPoly with those of other melamine phosphates suggested the protonation shown in Figure 1. The structure model obtained after the unrestrained refinement $\left(R_{\mathrm{wp}} \approx 0.09 ; R_{\mathrm{p}} \approx 0.068\right)$ was not completely satisfactory because of some minor distortions of bond length and bond angles, and therefore, it was decided to keep soft restraints $(f \approx 5)$. Details of the Rietveld refinement are given in Table 1; fractional coordinates and Uiso values of the final structure are listed in Table 2. Hydrogen bonds are listed in Table 3. Observed and calculated diffraction patterns show a good correspondence, even at high $2 \theta$ values (Figure 2).

Solid-State NMR Characterization. The single protonation of the melamines as discussed above is corroborated by solidstate NMR data. Figure $3 a-c$ shows a comparison of singlepulse ${ }^{1} \mathrm{H}$ MAS solid-state NMR spectra of MP, MPy, and MPoly, obtained at an external field of $18.8 \mathrm{~T}\left({ }^{1} \mathrm{H}\right.$ spectrometer frequency of $800 \mathrm{MHz}$ ), using $49 \mathrm{kHz}$ sample spinning to ensure spectral resolution.

Chemical shifts greater than $10 \mathrm{ppm}$ indicate protons involved in $\mathrm{R}-\mathrm{H} \cdots \mathrm{O}$ hydrogen bonding. ${ }^{37}$ Assignment of the ${ }^{1} \mathrm{H}$ chemical shifts in MP was done on the basis of ${ }^{1} \mathrm{H}-\mathrm{X}\left({ }^{31} \mathrm{P},{ }^{15} \mathrm{~N},{ }^{13} \mathrm{C}\right)$ HETCOR experiments as is discussed more extensively elsewhere. ${ }^{38}$ In the MP spectrum, the resonance at $13.7 \mathrm{ppm}$ stems from the $\mathrm{H}$-atom bonded to an endocyclic $\mathrm{N}$-atom, ${ }^{15,38}$ whereas the corresponding resonances in the MPy and MPoly spectra are only slightly shifted to 13.1 and $13.8 \mathrm{ppm}$, respectively. In
TABLE 2: Atomic Coordinates and Isotropic Displacement Parameters $U_{\text {iso }}\left(\right.$ in $\left.\AA^{2}\right)$ for MPoly

\begin{tabular}{lcccl}
\hline atom & $X$ & $Y$ & $Z$ & \multicolumn{1}{c}{$U_{\text {iso }}$} \\
\hline N1 & $-0.09877(15)$ & $2.3047(6)$ & $-0.04036(11)$ & $0.0327(11)$ \\
N2 & $0.01812(16)$ & $2.2611(7)$ & $0.16066(9)$ & $0.0327(11)$ \\
N3 & $-0.03635(26)$ & $2.2885(11)$ & $0.06085(23)$ & $0.0327(11)$ \\
N4 & $-0.1615(4)$ & $1.9954(14)$ & $0.02071(27)$ & $0.0327(11)$ \\
N5 & $-0.10231(25)$ & $1.9857(8)$ & $0.12706(10)$ & $0.0327(11)$ \\
N6 & $-0.22165(19)$ & $1.6898(8)$ & $0.08877(8)$ & $0.0327(11)$ \\
C7 & $-0.09808(30)$ & $2.2001(12)$ & $0.01631(24)$ & $0.0249(21)$ \\
C8 & $-0.04033(31)$ & $2.1798(12)$ & $0.11503(19)$ & $0.0249(21)$ \\
C9 & $-0.1608(4)$ & $1.8891(13)$ & $0.07945(20)$ & $0.0249(21)$ \\
P10 & $0.81017(18)$ & $-0.2339(8)$ & $0.27234(15)$ & $0.0256(9)$ \\
O11 & $0.73822(35)$ & $0.0190(14)$ & $0.27570(25)$ & $0.0189(12)$ \\
O12 & $0.82786(32)$ & $-0.3621(11)$ & $0.33363(22)$ & $0.0189(12)$ \\
O13 & $0.87966(30)$ & $-0.0829(12)$ & $0.24421(26)$ & $0.0189(12)$ \\
H14 & $-0.13965(22)$ & $2.2557(13)$ & $-0.06870(14)$ & 0.025 \\
H15 & $-0.06201(22)$ & $2.4388(8)$ & $-0.04733(18)$ & 0.025 \\
H16 & $0.0197(4)$ & $2.1751(11)$ & $0.19559(11)$ & 0.025 \\
H17 & $0.05914(20)$ & $2.3780(9)$ & $0.15413(19)$ & 0.025 \\
H18 & $-0.0980(4)$ & $1.8955(10)$ & $0.16182(11)$ & 0.025 \\
H19 & $-0.25914(24)$ & $1.6328(13)$ & $0.05879(12)$ & 0.025 \\
H20 & $-0.2211(4)$ & $1.6103(11)$ & $0.12420(10)$ & 0.025
\end{tabular}

TABLE 3: Hydrogen-Bonding Geometry in MPoly $\left(\AA{ }^{\circ}\right)$

\begin{tabular}{lllll}
\hline donor-H$\cdots$ acceptor $^{a}$ & $\mathrm{D}-\mathrm{H}$ & $\mathrm{H} \cdots \mathrm{A}$ & $\mathrm{D} \cdots \mathrm{A}$ & $\mathrm{D}-\mathrm{H} \cdots \mathrm{A}$ \\
$\mathrm{N} 1-\mathrm{H} 14 \cdots \mathrm{O} 12^{i}$ & $0.861(4)$ & $2.203(5)$ & $2.875(5)$ & $134.7(4)$ \\
$\mathrm{N} 2-\mathrm{H} 16 \cdots \mathrm{O} 13^{i i}$ & $0.860(4)$ & $2.229(7)$ & $2.884(6)$ & $132.9(6)$ \\
$\mathrm{N} 2-\mathrm{H} 17 \cdots \mathrm{O} 12^{i i i}$ & $0.860(4)$ & $2.112(6)$ & $2.943(6)$ & $162.5(4)$ \\
$\mathrm{N} 5-\mathrm{H} 18 \cdots \mathrm{O} 13^{i v}$ & $0.861(4)$ & $1.923(7)$ & $2.682(7)$ & $146.4(5)$ \\
$\mathrm{N} 6-\mathrm{H} 19 \cdots \mathrm{N}^{v}$ & $0.860(4)$ & $2.084(7)$ & $2.939(7)$ & $172.9(4)$ \\
$\mathrm{N} 6-\mathrm{H} 20 \cdots \mathrm{O} 11^{i i}$ & $0.860(4)$ & $2.320(7)$ & $3.156(7)$ & $164.4(5)$
\end{tabular}

${ }^{a}$ Symmetry codes: ${ }^{i}-1+\mathrm{x}, 2-\mathrm{y},-1 / 2+\mathrm{z} \cdot{ }^{i i} 1-\mathrm{x}, 2+\mathrm{y}, 1 / 2-$ z. ${ }^{i i i} 1-\mathrm{x}, 3+\mathrm{y},{ }^{1 / 2}-$ z. $^{i v}-1+\mathrm{x}, 2+\mathrm{y}, \mathrm{z} \cdot{ }^{v}-1 / 2-\mathrm{x},{ }^{7 / 2}-\mathrm{y},-\mathrm{z}$. $v i 1 / 2-\mathrm{x}, 3 / 2+\mathrm{y}, 1 / 2-\mathrm{z}$.

the MP spectrum, the resonances at 11.1 and $13.2 \mathrm{ppm}$ result from the $\mathrm{H}$-atoms bonded to the phosphate oxygen atoms. Because of the condensation of the $\mathrm{PO}_{4}$ groups, only one resonance of this type remains in the MPy spectrum, and finally, none remains in the MPoly spectrum. The resonances of the exocyclic $\mathrm{NH}_{2}$ groups appear in all spectra (MP, MPy, and MPoly) in the chemical shift range 4-10 ppm, where the spectral differences support a change in the melamine stacking and bonding going from MP to MPy to MPoly. More detailed conclusions cannot be drawn, however. Density functional theory (DFT) calculations of proton chemical shifts could provide a deeper insight and are under investigation.

Figure 4 shows single-pulse ${ }^{31} \mathrm{P}$ spectra of two MPoly samples from the same batch for comparison. The resonance at -25.5 ppm (marked "mid" in Figure 4c) stems from the $\mathrm{PO}_{3}$ 


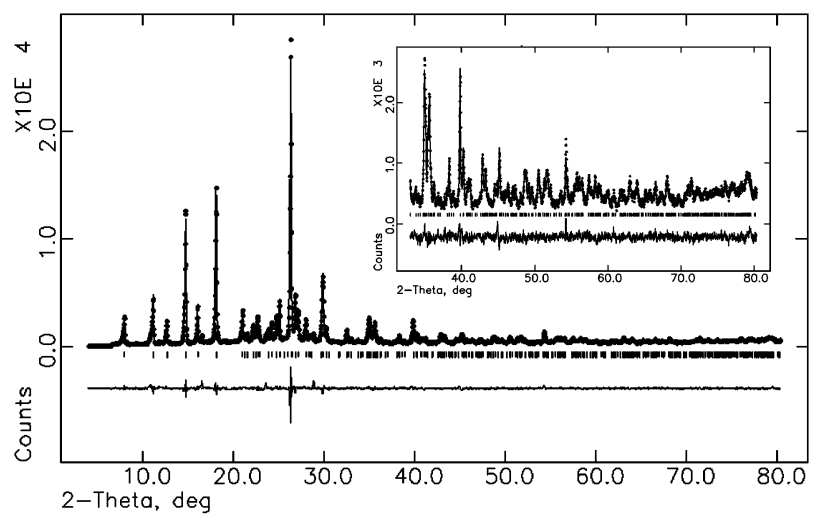

Figure 2. Full range and high-angle $\mathrm{X}$-ray powder diffraction patterns of MPoly, showing the experimental pattern (dots), the final calculated pattern after refinement (line), the difference (experimental - calculated) pattern (line at the bottom), and the positions of the reflections (vertical lines).

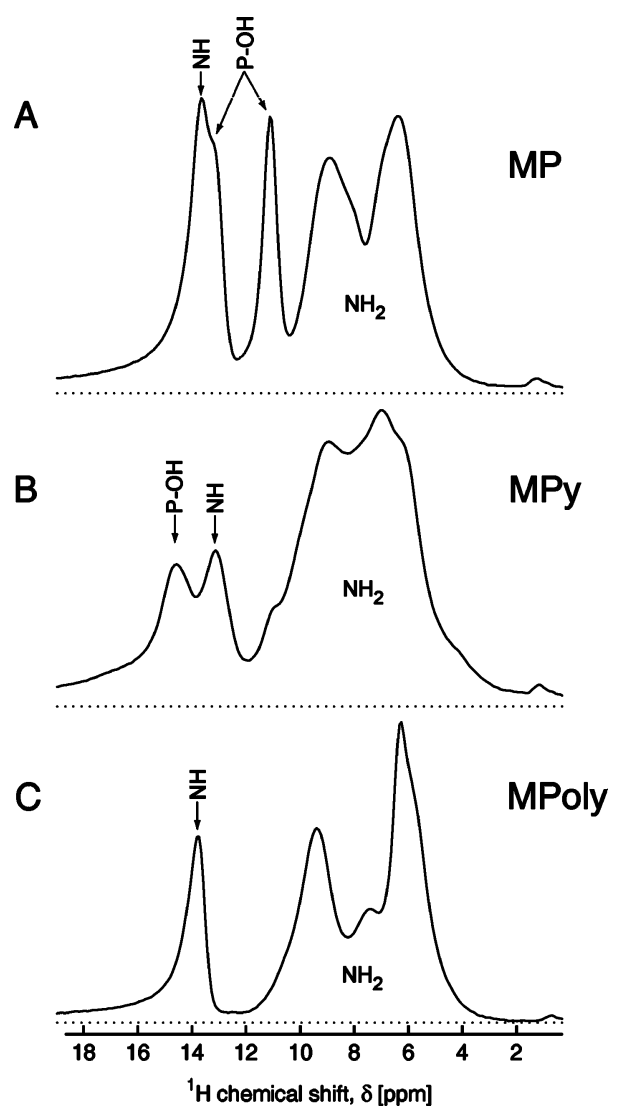

Figure 3. (a) Single-pulse ${ }^{1} \mathrm{H}$ MAS solid-state NMR spectrum of MP. (b, c) Corresponding spectra of MPy and MPoly, respectively.

groups in the middle of the polyphosphate chain in MPoly. The splitting results from the $n=0$ rotational resonance between adjacent phosphate units. The small resonance at $-12.2 \mathrm{ppm}$ (marked "end") originates from the chain ends of the polyphosphate chain or pyrophosphate impurities. The chain lengths for these two samples are estimated therefrom to be at least $\sim 105$ and $\sim 170$, respectively, indicating a variation in average chain lengths in different samples from the same batch. But in all other cases, the average chain length was also established to be larger than $\sim 100$.

The resonances in the range -2 to $2 \mathrm{ppm}$ in the ${ }^{31} \mathrm{P}$ spectrum stem from orthophosphates present in the sample. Figure $4 a, b$ shows this spectral range for two MPoly samples in closer detail. At least two components at 0 and $-1.2 \mathrm{ppm}$ can be identified

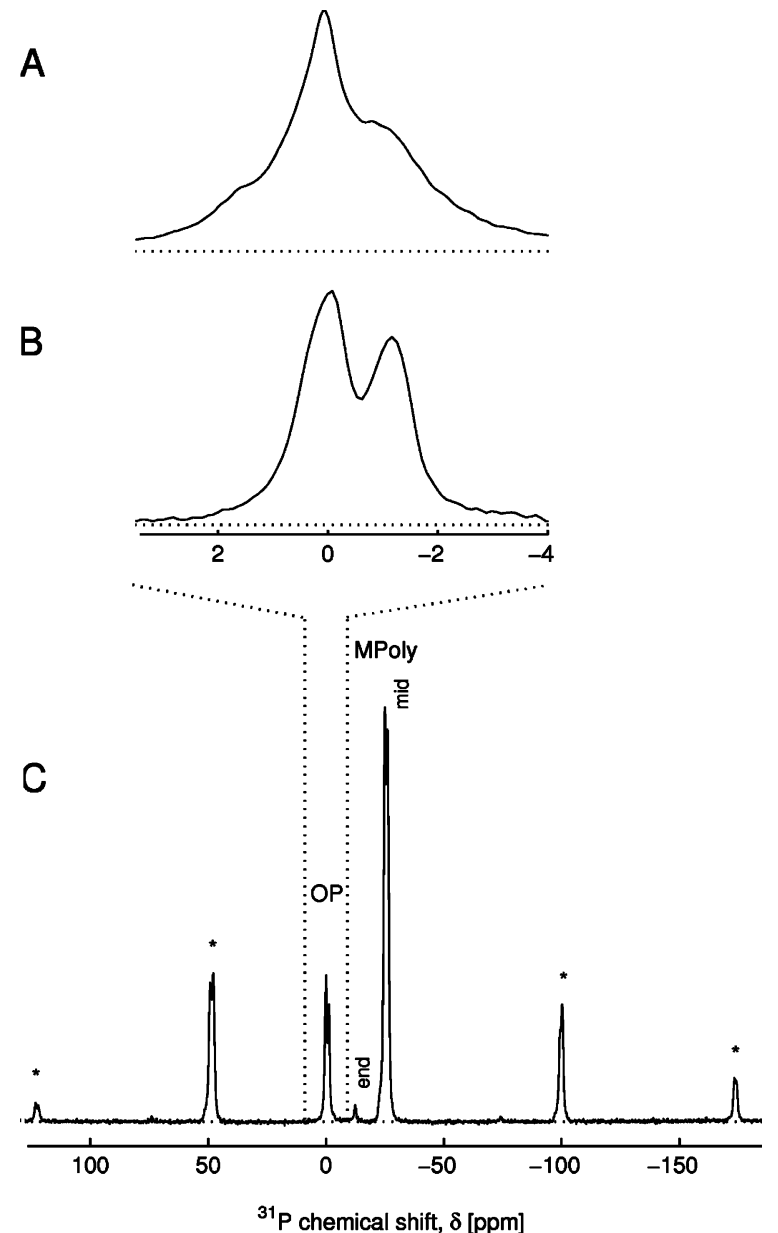

Figure 4. Single-pulse ${ }^{31} \mathrm{P}$ MAS solid-state NMR spectra of two MPoly samples from the same batch: (a) first sample; (b, c) second sample. (a) and (b) show enlarged spectra of the orthophosphate (OP) region $(-2$ to $2 \mathrm{ppm})$ in the MPoly spectrum, whereas (c) shows a complete MPoly spectrum. Side bands are labeled with asterisks (*). For the interpretation of "mid" and "end", see text.

whose relative intensities vary with respect to each other and with respect to the MPoly resonance in the two samples. The amount of orthophosphates present is estimated to $14 \%$ and $24 \%$ for the two samples, respectively. It should be noted that the component at $-1.2 \mathrm{ppm}$ is not present in CP-MAS spectra (Figure 5), suggesting high mobility of this orthophosphate component.

Figure 6a shows the experimental results of a ${ }^{31} \mathrm{P}$ saturation recovery experiment for the polyphosphate resonance in MPoly. The ${ }^{31} \mathrm{P}$ signal intensity as a function of the recovery time $\tau$ shows strong nonexponential behavior, that is, is described by a distribution of $T_{1}$ relaxation time constants. Therefore, a Kohlrausch-Williams-Watts stretched exponential function ${ }^{39,40}$ was chosen to fit the data

$$
f(\tau)=a\left\{1-\exp \left[-\left(\tau / \tilde{T}_{1}\right)^{\beta}\right]\right\}
$$

The parameter $\tilde{T}_{1}$ is the time constant for the nonexponential longitudinal relaxation, $\beta$ is the stretch parameter (with $0 \leq \beta$ $\leq 1$ ), and $a$ is an overall scaling constant. It is possible to obtain the average value of the longitudinal relaxation time constant, $\left\langle T_{1}\right\rangle$, without knowing the distribution function of the individual $T_{1}$ using 40

$$
\left\langle T_{1}\right\rangle=\frac{\tilde{T}_{1}}{\beta} \Gamma(1 / \beta)
$$




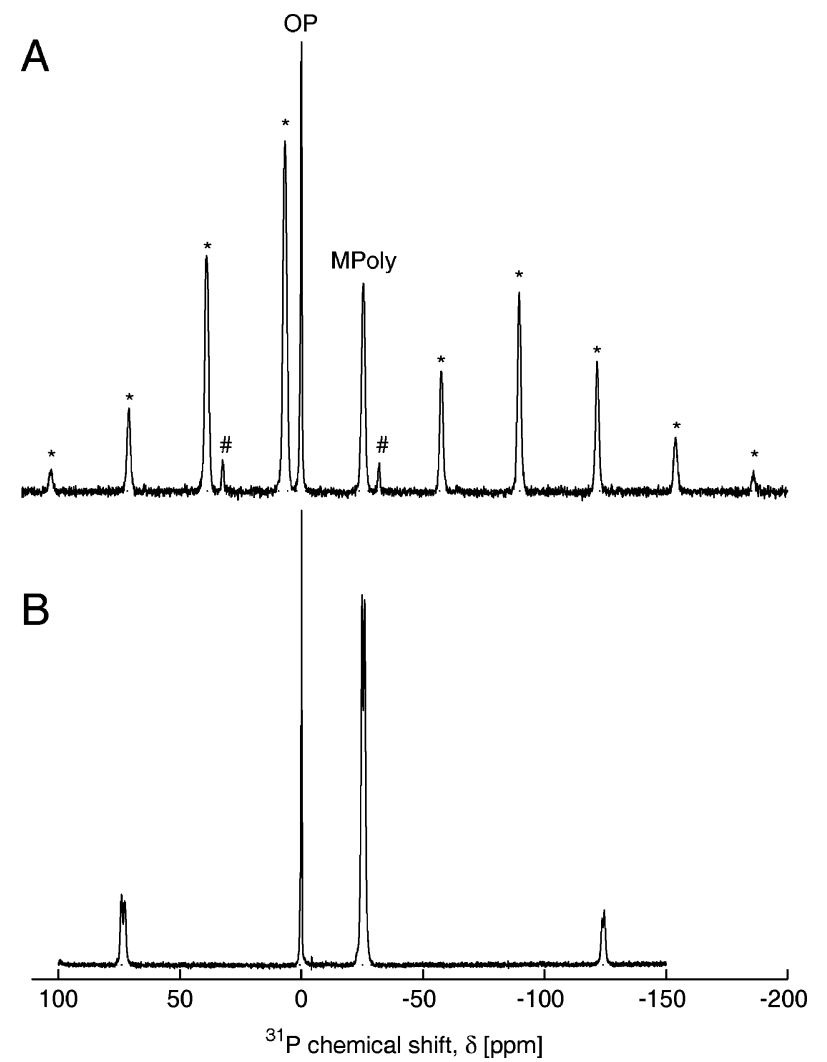

Figure 5. ${ }^{31} \mathrm{P}$ CP-MAS spectra of MPoly at a spinning frequency of (a) $4 \mathrm{kHz}$ and (b) $12 \mathrm{kHz}$. In (a), the center band and side bands of the phosphorus sites in MPoly are labeled (MPoly) and (*), respectively. In addition, the sample contains a fraction of an orthophosphate compound, for which the center band and side bands are labeled (OP) and (\#), respectively.

where $\Gamma(\alpha)$ is the gamma function. ${ }^{41}$ We obtained a value of $\left\langle T_{1}\right\rangle=837 \pm 24 \mathrm{~s}$. The stretch parameter $\beta$ is given by $0.75 \pm$ 0.01 , which is most likely caused by the spread in chain lengths in the MPoly sample.

Figure $6 \mathrm{~b}, \mathrm{c}$ shows the experimental results of saturation recovery experiments for the orthophosphate components in MPoly and of MP, respectively. The experimental data in Figure $6 \mathrm{~b}$ shows clear evidence of multiexponential behavior. Neither a single stretched exponential function nor a sum of two exponential functions could describe it satisfactorily. The model with the minumum number of parameters that described the data adequately consisted of a sum of an exponential and a stretched exponential function, as indicated in the figure caption, resulting in longitudinal relaxation times of $T_{1}^{(1)}=0.30 \pm 0.03$ $\mathrm{s}$ and $\left\langle T_{1}{ }^{(2)}\right\rangle=538 \pm 91 \mathrm{~s}$ for the orthophosphate components in MPoly. As a comparison, in Figure 6c the results for MP are shown, which could be described satisfactorily using a singleexponential function, resulting in a $T_{1}$ value of $1362 \pm 60 \mathrm{~s}$. This is considerably larger than for the orthosphosphate components in MPoly. In addition, the two-dimensional ${ }^{1} \mathrm{H}-$ ${ }^{31} \mathrm{P}$ heteronuclear correlation spectrum of MPoly (Figure 7b) shows cross-peaks of the ${ }^{31} \mathrm{P}$ orthophosphate resonance at $0 \mathrm{ppm}$ with two relatively sharp proton resonances at 6.4 and $14.2 \mathrm{ppm}$, where the latter indicates hydrogen-bonded protons. As a comparison, Figure 7c shows the corresponding slice of a twodimensional ${ }^{1} \mathrm{H}-{ }^{31} \mathrm{P}$ heteronuclear correlation spectrum of MP. The conclusion is that the orthophosphate components in the MPoly samples do not consist of MP. We attribute the orthophosphate resonance at $0 \mathrm{ppm}$ to a crystalline phase of orthophosphate(s) that is subjected to hydrogen bonding, whereas the resonance at $-1.2 \mathrm{ppm}$ is attributed to a noncrystal-


Figure 6. ${ }^{31} \mathrm{P}$ single-pulse signal intensities as a function of the recovery time $\tau$ in a saturation recovery experiment for (a) the polyphosphate sites in MPoly and the orthophosphate sites in (b) MPoly and (c) MP. Symbols: integrated experimental spectral intensities. Solid lines: best fit results to different build-up functions. (a) KohlrauschWilliams-Watts stretched exponential as defined in eq 1 with $\left(\tilde{T}_{1}, \beta\right)$ $=(704 \pm 18 \mathrm{~s}, 0.75 \pm 0.01)$. (b) Sum of an exponential and a stretched exponential function: $f(\tau)=a\left(1-\exp \left\{-\tau / T_{1}{ }^{(1)}\right\}\right)+(1-a)(1-$ $\left.\exp \left\{-\left(\tau / \tilde{T}_{1}{ }^{(2)}\right)^{\beta}\right\}\right)$ with $a=0.35 \pm 0.02, T_{1}^{(1)}=0.30 \pm 0.03 \mathrm{~s}, \widetilde{T}_{1}^{(2)}=$ $374 \pm 46 \mathrm{~s}$, and $\beta=0.62 \pm 0.06$.

line phase of orthophosphate(s) with a higher mobility. Both orthophosphate phase components could stem from phosphoric acid (crystalline and noncrystalline), ${ }^{42}$ for example, in inclusions or at the surface of the particles. The analysis of the XRPD patterns did not allow an attribution of the non-MPoly peaks to any known orthophosphate phase, suggesting the presence of some unknown melamine orthophosphate salt(s).

Reheating the first sample to about $250{ }^{\circ} \mathrm{C}$ and $280{ }^{\circ} \mathrm{C}$ for about $4 \mathrm{~h}$ did not produce more orthophosphates, but nearly all of the orthophosphate impurities condensed. In the ${ }^{31} \mathrm{P}$ singlepulse NMR spectrum, they showed up as a broad resonance pattern around $-12 \mathrm{ppm}$, but different from the chain-end resonance patterns of MPy and MPoly. A degradation of MPoly into orthophosphates was not observed.

Crystal Structure of MPoly and Its Comparison with Related Structures. The MPoly crystal structure consists of layers of "infinite" melamine cation chains alternating with layers of polyphosphate anion chains (Figure 8). Within the melamine chains, adjacent moieties are linked by means of sideby-side pairs of $\mathrm{N}-\mathrm{H} \cdots \mathrm{N}$ hydrogen bonds $(\mathrm{N} \cdots \mathrm{N} \approx 2.89 \AA)$ 




Figure 7. (a) Single-pulse ${ }^{1} \mathrm{H}$ MAS solid-state NMR spectrum of MPoly. (b) Two-dimensional ${ }^{1} \mathrm{H}-{ }^{31} \mathrm{P}$ heteronuclear correlation spectrum of MPoly and integrated slices of the MPoly (upper slice) and orthophosphate ${ }^{31} \mathrm{P}$ (lower slice) resonances. (c) Integrated slice of the two-dimensional ${ }^{1} \mathrm{H}-{ }^{31} \mathrm{P}$ heteronuclear correlation spectrum of MP.

forming infinite ribbons with all molecules being parallel and lying approximately in one plane $(\sigma=\sim 0.1 \AA$ ), a type of packing commonly found for melamine complexes and salts. ${ }^{16}$ The ribbons within one layer are parallel, while the angle between the planes of melamine molecules in two neighboring layers is $81.4^{\circ}$. The stacking distance between melamine ribbons (3.31 $\AA$ ) is shorter than the usual distance between $\pi$-aromatic ring systems $(\sim 3.4 \AA)^{43}$ and indicates $\pi-\pi$ interactions between the melamines.

Melamine's internal $\mathrm{C}-\mathrm{N}-\mathrm{C}$ angle at the protonated endocyclic nitrogen $(\mathrm{N} 5)$ is significantly larger $\left(\sim 118^{\circ}\right)$ than the other two $\mathrm{C}-\mathrm{N}-\mathrm{C}$ angles (mean $\sim 115^{\circ}$ ). This difference is characteristic for all known melamine salts with single-protonated melamine moieties ${ }^{15,16,44}$ and has been corroborated by densityfunctional geometry optimization of a single protonated melamine molecule. ${ }^{44}$ The angular differences are due to the steric effect of the lone-pair electron, as predicted by the valence-shell electron-pair repulsion theory (VSEPR). ${ }^{45}$

The melamine ribbons are linked by polyphosphates running parallel to the $b$ axis. Rather typical for melamine phosphates, ${ }^{15,16,44}$ one of the two terminal oxygens of each $\mathrm{PO}_{3}$ group participates in an $\mathrm{O} \cdots \mathrm{H}-\mathrm{N}^{+}$ionic hydrogen bond with an endocyclic nitrogen of the neighboring melamine moiety, while the oxygen in the $\mathrm{P}-\mathrm{O}-\mathrm{P}$ bridge forms a second hydrogen bond with an $\mathrm{NH}_{2}$ group of the same melamine (Figure 9). Three other melamine moieties bind via $\mathrm{NH}_{2}$ groups to the remaining terminal oxygens of $\mathrm{PO}_{3}$ groups, and in this way, each oxygen participates in at least one hydrogen bond, although as the chemical shifts of these protons are below $10 \mathrm{ppm}$, these $\mathrm{N}-\mathrm{H}_{2} \cdots \mathrm{O}$ hydrogen bonds must be very weak.

Polyphosphate chains can adapt their configuration quite easily because of a high flexibility of the constituent fragments and can readily meet any geometric and coordination requirements set by cations. (see refs 25-31 and 46 and references therein). Nevertheless, bond lengths and angles are usually similar in polyphosphate configurations. In MPoly, the usual two types of $\mathrm{P}-\mathrm{O}$ bonds can be observed: The largest distances
(1.60-1.63 $\AA$ ) are related to the $\mathrm{P}-\mathrm{O}-\mathrm{P}$ linkage within the chain, and the shortest distances $(1.46-1.50 \AA)$ characterize $\mathrm{P}-\mathrm{O}$ bonds involving terminal oxygens. Angular ranges for $\mathrm{O}-\mathrm{P}-\mathrm{O}\left(102.7-120.6^{\circ}\right)$ and $\mathrm{P}-\mathrm{O}-\mathrm{P}\left(134.2^{\circ}\right)$ angles are also in a good agreement with those usually found in polyphosphate anions. ${ }^{25-31,46}$

Figure 5 shows the ${ }^{31} \mathrm{P}$ CP MAS spectra of MPoly at two different sample spinning frequencies, (a) $4 \mathrm{kHz}$ and (b) $12 \mathrm{kHz}$. A Herzfeld-Berger analysis ${ }^{47}$ of the sideband pattern in Figure 6a for the phosphorus $\mathrm{PO}_{3}$ sites in MPoly results in a chemical shift anisotropy $(\mathrm{CSA})$ of $\left(\delta_{11}, \delta_{22}, \delta_{33}\right)=(78,17,-172) \pm 1$ ppm, which is in good agreement with literature data for $\mathrm{PO}_{3}$ sites in polyphosphate salts. ${ }^{48}$ Although all phosphorus nuclei inside the polyphosphate chain have identical chemical shift tensors, the relative orientation of the CSA tensors of two adjacent phosphorus sites with respect to the interconnecting dipolar coupling vector is different. If the two chemical shift tensors are related by a symmetry operation other than inversion symmetry, the averaging of the dipolar interaction under MAS is effectively prevented, referred to as $n=0$ rotational resonance. ${ }^{49,50}$ This effect has been used to study CSA tensors and dipolar and $J$ couplings in isolated ${ }^{31} \mathrm{P}-{ }^{31} \mathrm{P}$ spin pairs. ${ }^{51,52}$ As a result of the $n=0$ rotational resonance between two adjacent phosphorus sites inside the polyphosphate chain, a splitting can be observed in the MPoly spectrum in Figure 6b, obtained at $12 \mathrm{kHz}$ spinning, which is due to the homonuclear dipolar coupling. Numerical simulation of the ${ }^{31} \mathrm{P}$ spectrum of the two middle phosphates in a tetraphosphate fragment of the polyphosphate chain in MPoly led to results (not shown) that are compatible with the presented geometry of the polyphosphate chain.

There are many known polyphosphate structures (see refs 2531 and 46 and references therein). Ammonium polyphosphate ${ }^{46}$ is also used in flame-retardant applications, ${ }^{3}$ and its polyphosphate chains are rather similar to those in MPoly (RMS $\approx$ $0.2 \AA$ ). This suggests that, to some extent, exchange or mixing of (similar) cations might be possible, without affecting the polyphosphate chain packing. In this respect, it is interesting to note that recently crystals of an ammonium-melamine copolyphosphate were synthesized and claimed to have superior thermal stability during the processing of polymers compared to the pure components. ${ }^{53}$

Structural Changes along the Condensation Route MP $\rightarrow$ MPy $\rightarrow$ MPoly. In many aspects, the crystal structure of MPoly resembles those of MP and MPy. All three compounds have a layered structure in which layers of melamine ribbons and phosphate chains alternate (see Figure 8). The type of melamine ribbons ${ }^{16}$ and the character of the melamine-tophosphate bonding also remains the same along the condensation route. Still, some significant differences exist between MPoly and its precursors MP and MPy. In the latter two, (pyro)phosphate chains are paired, while MPoly has only a single polyphosphate chain (Figure 10). Apparently, the condensation MPy $\rightarrow$ MPoly is not a simple intrachain process, as in MP $\rightarrow$ MPy, ${ }^{16}$ but a significant rearrangement of phosphate groups and melamine packing is required: the melamines reorient to retain their bonding to the phosphates as discussed earlier in more detail. As a result, in MPoly not all the melamines are parallel to each other, as in MP and MPy, but they are arranged in two sets of parallel planes with a large interangle (Figure 8a).

An analysis of the crystal structures of MPoly and MPy points out why the polymerization is not a simple intrachain process. The closest distance between two phosphorus atoms in two neighboring pyrophosphates in the same phosphate chain is 


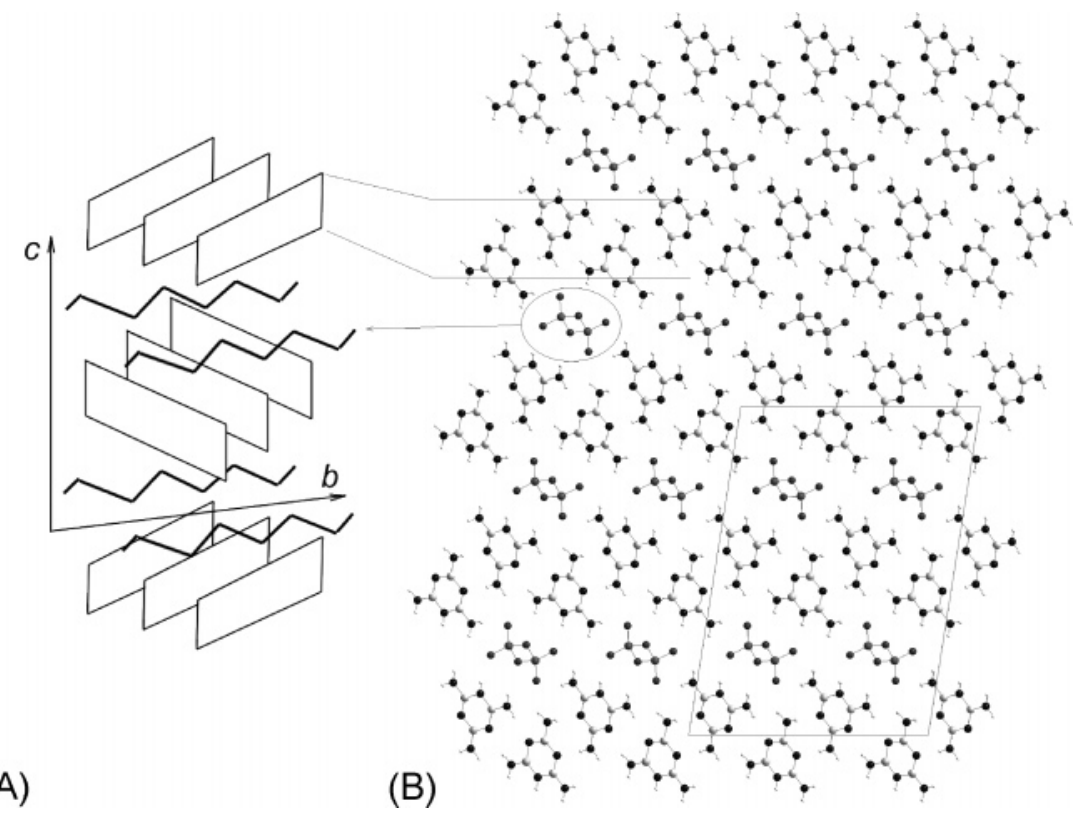

Figure 8. Schematic representation of the crystal packing of MPoly (A) and its projection on the plane perpendicular to phosphate chain direction (B).



Figure 9. Hydrogen bonds between melamines and $\mathrm{PO}_{3}$ group of the polyphosphate moiety in MPoly. For the hydrogen bond distances, see Table 3.

$\sim 4.3 \AA$ (Figure 11). On the other hand, the phosphor-phosphor distance required to form a $\mathrm{P}-\mathrm{O}-\mathrm{P}$ bridge is about $3.0 \AA \AA^{25-31}$ Thus, a simple intrachain polymerization, as in MP $\rightarrow$ MPy, would require an additional decrease of the distance between the melamine layers, assuming that the melamine-phosphate bonding character remains the same. This is unlikely to happen, because the short distance between the melamine layers in MPy ( $\sim 3.25 \AA$ ) already implies quite strong $\pi-\pi$ interactions, and a further (large) decrease will lead to repulsion between the layers. As it turns out, the melamine-stacking distance in MPoly $(\sim 3.31 \AA)$ is larger than in MPy $(\sim 3.25 \AA)$. It is noted that the change of the melamine-stacking distance, $\sim 3.42,3.25$, and $3.31 \AA$ in MP, MPy, and MPoly, respectively, shows the same trend as the change in density of the compounds $(1.71,1.81$, and $1.78 \mathrm{~g} \mathrm{~cm}^{-3}$ ), although this may be a coincidence.

Method of MPoly Preparation and Its Structural Characteristics. Recently, it has been claimed that the method of melamine-polyphosphate production can influence the thermal stability of the product. ${ }^{4,5}$ Given the large variation found in both the degree of polymerization and the orthophosphate impurity level within the same batch, the precise processing conditions may well be influential. 


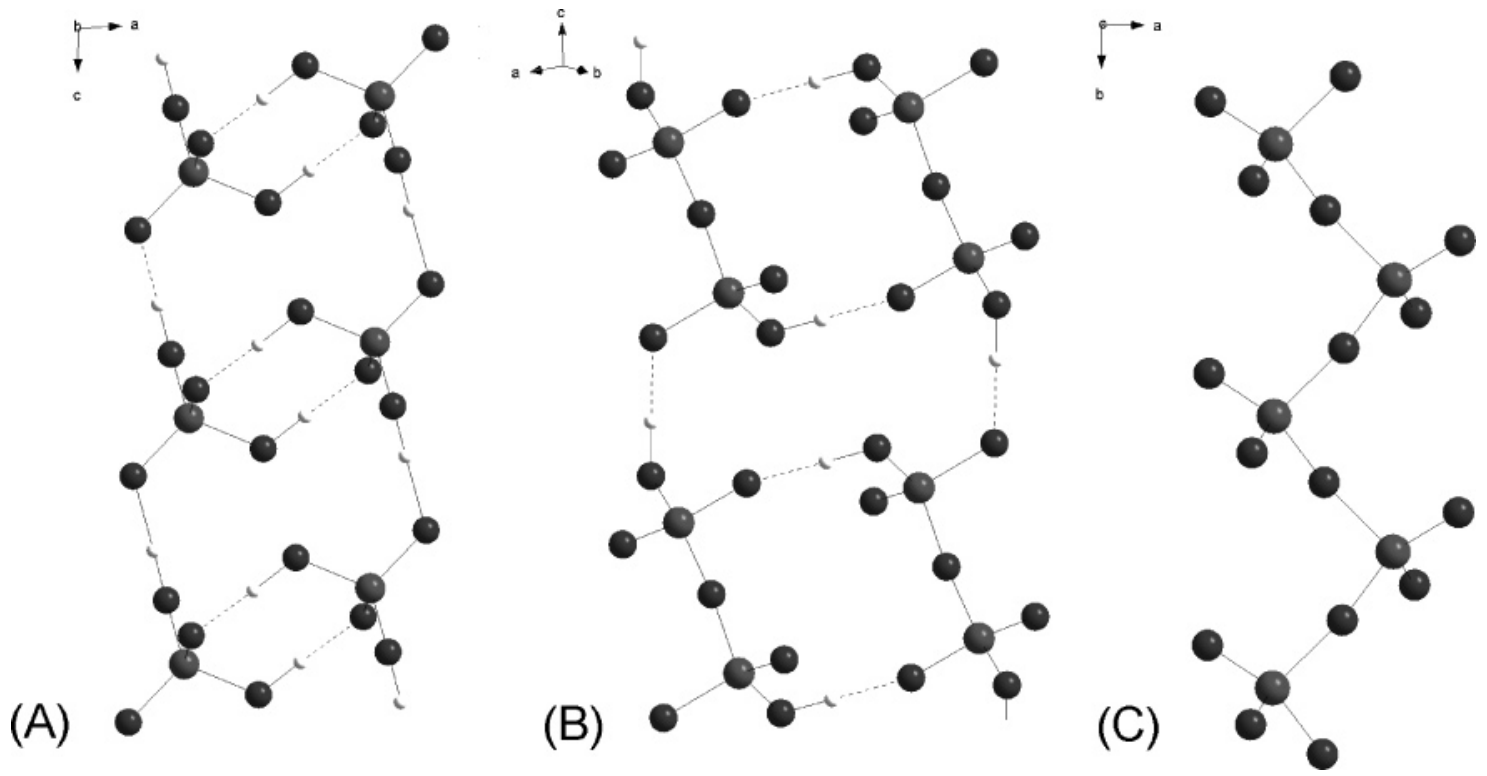

Figure 10. Pairs of phosphate chains in MP (A), pairs of pyrophosphate chains in MPy (B) and an infinite chain of phosphates in MPoly (C).

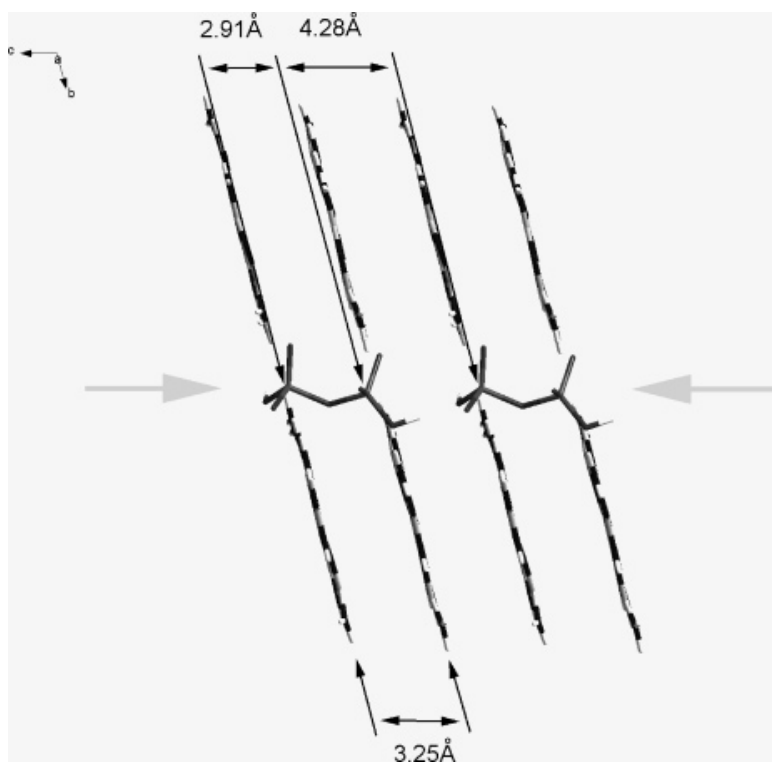

Figure 11. The crystal structure of MPy with the $a$ axis perpendicular to the plane of the paper. The distances between melamine layers $(3.25 \AA)$, the distance between two closest phosphorus atoms in two neighboring pyrophosphates $(4.28 \AA)$, and the distance between two phosphorus atoms of one pyrophosphate moiety $(2.91 \AA)$ are marked. The two arrows indicate the direction of melamine layer compression required in the case of intrachain polymerization.

From this point of view, and in order to rationalize the synthesis of the melamine polyphosphate MPoly considered here, it is important to know if, starting from MP, it would be possible to obtain other types of polymetaphosphates by different preparation techniques. Crystal-structure analysis suggests that this is not likely: Sets of two (pyro)phosphate chains in MP (and MPy) hardly interact, and polymerization can proceed only within a set (inter- or intrachain), resulting in linear polymethaphosphates with a predefined direction.

\section{Summary}

Upon condensation of MP to MPoly (via MPy), the phosphates form layers of polyphosphate anion chains. The melamine cations form hydrogen-bonded ribbons with all molecules being parallel within one layer. The $\pi-\pi$ interactions between the melamines in MPoly are stronger than in MP and MPy, as indicated by the shortened melamine-ribbon packing distance. The melamine ribbons are linked to the polyphosphates through $\mathrm{O}-\mathrm{HN}$ hydrogen bonds. The condensation MPy $\rightarrow$ MPoly is not a simple intrachain process, as in MP $\rightarrow$ MPy, but a significant rearrangement of phosphate groups and melamine packing is required. The average chain length was established to be larger than $\sim 100$, but large variations do occur within the same batch. Furthermore, a substantial fraction of the samples turned out to consist of orthophosphate impurities that differ from MP. Hence, the MPoly batch and its samples are inhomogeneous at the structural level (chain length) as well as at the compositional level (impurities). Nevertheless, a crystal structure determination of MPoly from XRPD data could still be carried out.

Acknowledgment. The authors acknowledge E. J. Sonneveld for his help in data collection and indexing. They also thank Dr. R. B. Helmholdt, Dr. D. J. A. De Ridder, Dr. V. M. Litvinov, Dr. B. Coussens, Dr. A. Braam, and Dr. K. Goubitz for useful discussions, and DSM for the sample preparation and the density measurements of MPoly. This work was supported by DSM (Geleen, The Netherlands), Ciba Speciality Chemicals (Basel, Switzerland), and The Netherlands Foundation for Scientific Research (NWO).

\section{References and Notes}

(1) Jahromi, Sh.; Gabriëlse, W.; Braam, A. Polymer 2003, 44, 25.

(2) Cichy, B.; Luczkowska, D.; Nowak, M.; Wladya-Przybylak, M. Ind. Eng. Chem. Res. 2003, 42, 2897.

(3) Weil, E.; McSwigan, B. J. Coat. Technol. (Tokyo) 1994, 66, 75.

(4) Suzuki, K.; Shishido, K.; Shindo, M. U.S. Patent 6,008,349, 1999.

(5) Kersjes, J. G.; Kierkels, R. H. M. (a) Patent PCT/NL/99/00426, 1999; (b) Patent WO 00/02869, 2000; (c) U.S. Patent 6,653,474 B1, 2003.

(6) Horacek, H.; Pieh, S. Polym. Int. 2000, 49, 1106.

(7) Chen, W. Y.; Wang, Y. Z.; Chang, F. C. J. Appl. Polym. Sci. 2004, 92(2) 892

(8) Kasowski, R. V. U.S. Patent 6,268,494 B1, 2001.

(9) Zhu, W.; Weil, E. D.; Mukhopadhyay, S. J. Appl. Polym. Sci. 1996, 62, 2267.

(10) Harris, K. D. M. Cryst. Growth Des. 2003, 3(6), 887.

(11) Schnell, I.; Brown, S. P.; Low, H. Y.; Ishida, H.; Spiess, H. W. J. Am. Chem. Soc. 1998, 120(45), 11784.

(12) Brown, S. P.; Spiess, H. W. Chem. Rev. 2001, 101(12), 4125.

(13) Goward, G. R.; Schnell, I.; Brown, S. P.; Spiess, H. W.; Kim, H.D.; Ishida, H. Magn. Reson. Chem. 2001, 39(S1), S5. 
(14) Goward, G. R.; Sebastiani, D.; Schnell, I.; Spiess, H. W.; Kim, H.-D.; Ishida, H. J. Am. Chem. Soc. 2003, 125(19), 5792.

(15) De Ridder, D. J. A.; Goubitz, K.; Brodski, V.; Peschar, R.; Schenk, H. Helv. Chim. Acta 2004, 87, 1894-1904.

(16) Brodski, V.; Peschar, R.; Schenk, H.; Brinkmann, A.; van Eck, E. R. H.; Kentgens, A. P. M., Coussens, B.; Braam, A. J. Phys. Chem. 2004, 108, 15069-15076.

(17) Roberts, B.; Parrish, W. International Tables for Crystallography, Vol. III; MacGillavry, C. H., Rieck, G. D., Eds.; Kynoch Press: Birmingham, 1962; pp 73-88.

(18) Visser, J. W. J. Appl. Crystallogr. 1969, 2, 89.

(19) Brodski, V.; Peschar, R.; Schenk, H. J. Appl. Crystallogr. 2003, $36,239$.

(20) Larson, A. C.; Von Dreele, R. B. General Structure Analysis System $(G S A S)$; Report LAUR 86-748, Los Alamos National Laboratory, Los Alamos, NM 87545; U.S. Government Printing Office: Washington, DC, 1994.

(21) Van Laar, B.; Yelon, W. B. J. Appl. Crystallogr. 1984, 17, 47.

(22) Finger, L. W.; Cox, D. E.; Jephcoat, A. P. J. Appl. Crystallogr. 1994, 27, 892 .

(23) Von Dreele, R. B. J. Appl. Crystallogr. 1997, 30, 517.

(24) Nowell, H.; Attfield, J. P.; Cole, J. C. Acta Crystallogr., Sect. B 2002, 58, 835 .

(25) Jaouadi, K.; Naili, H.; Zouari, N.; Mhiri, T.; Daoud, A. J. Alloys Compd. 2003, 354, 104.

(26) El Abiad, A. E.; Es-Sakhi, B.; Mesnaoui, M.; Maazaz, M.; Belharouak, I.; Gravereau, P.; Parent, C.; Wallez, G.; Le Flem, G. J. Solid State Chem. 2000, 154, 584.

(27) Graia, M.; Driss, A.; Jouini, T. Solid State Sci. 2003, 5, 393.

(28) Aouad, H.; Maazaz, M.; Belharouak, I. Mater. Res. Bull. 2000, $35,2457$.

(29) Murashova, E. V.; Chudinova, N. N. Cryst. Rep. 2001, 46(6), 942.

(30) Murashova, E. V.; Chudinova, N. N. Inorg. Mater. 2000, 36(12), 1512.

(31) Bruhne, B.; Jansen, M. Z. Anorg. Allg. Chem. 1994, 620(5), 931.

(32) Allen, F. H.; Kennard, O. Chem. Des. Autom. News 1993, 8, 31.
(33) Samoson, A. In Encyclopedia of NMR; 2002; Vol. 9 (Advances in NMR), p 59.

(34) Bennett, A. E.; Rienstra, C. M.; Auger, M.; Lakshmi, K. V.; Griffin, R. G. J. Chem. Phys. 1995, 103, 6951.

(35) Bielecki, A.; Kolbert, A. C.; Levitt, M. H. Chem. Phys. Lett. 1989, 155,341 .

(36) Ramamoorthy, A.; Wu, C. H.; Opella, S. J. J. Magn. Reson. 1999, $140,131$.

(37) Harris, R. K.; Jackson, P.; Merwin, L. H.; Say, B. J.; Hagele, G. J. Chem. Soc., Faraday Trans. 1988, 84, 3649.

(38) Brinkmann, A.; van Eck, E. R. H.; Kentgens, A. P. M. In manuscript.

(39) Narayanan, A.; Hartman, J. S.; Bain, A. D. J. Magn. Reson., Ser. A 1995, 112, 58 .

(40) Laviolette, M.; Auger, M.; Désilets, S. Macromolecules 1999, 32, 1602 .

(41) Mathews, J.; Walker, R. L. Mathematical Methods of Physics; Addison-Wesley: New York, 1970.

(42) Blessing, R. H. Acta Crystallogr., Sect. B 1988, 44, 334.

(43) Pauling, L. The Nature of the Chemical Bond, 3rd ed.; Cornell University Press: Ithaca, NY, 1960; p 262.

(44) Janczak, J.; Perpétuo, G. J. Acta Crystallogr., Sect C 2002, 58 , 455 .

(45) Gillespie R. J. (a) J. Chem. Educ. 1963, 40, 295; (b) Chem. Soc. Rev. 1992, 21, 59.

(46) Bagiec-Beucher, M.; Guitel, J.-C. Z. Anorg. Chem. 1988, 559, 123.

(47) Herzfeld, J.; Berger, A. E. J. Chem. Phys. 1980, 73, 6021.

(48) Duncan, T. M. A Compilation of Chemical Shift Anisotropies; Farragut Press: Chicago, 1990.

(49) Maricq, M. M.; Waugh, J. S. J. Chem. Phys. 1979, 70, 3300.

(50) Dusold, S.; Sebald, A. Annu. Rep. NMR Spectrosc. 2000, 41, 185.

(51) Dusold, S.; Klaus, E.; Sebald, A.; Bak, M.; Nielsen, N. C. J. Am. Chem. Soc. 1997, 119, 7121.

(52) Dusold, S.; Milius, W.; Sebald, A. J. Magn. Reson. 1998, 135, 500.

(53) Kierkels, R. H. M.; Aelmans, N. J. J.; Grolleman, P. H. C.; Braam, A. W. M. Int. Pat. Appl. WO 03/031417 A1, 2003. 